\title{
Best neural simultaneous approximation
}

\author{
Hawraa Abbas Almurieb, Eman S. Bhaya \\ Department of Mathematics, College of Education for Pure Sciences, University of Babylon, Iraq
}

\begin{tabular}{l}
\hline Article Info \\
\hline Article history: \\
Received Jan 14, 2020 \\
Revised Apr 28, 2020 \\
Accepted Jun 2, 2020 \\
\hline
\end{tabular}

\section{Keywords:}

2-normed space

Approximation

Neural networks

\begin{abstract}
For many years, approximation concepts has been investigated in view of neural networks for the several applications of the two topics. Researchers studied simultaneous approximation in the 2-normed space and proved essential theorems concern with existence, uniqueness and degree of best approximation. Here, we define a new 2 -norm in $L p$-space, with $p<1$, so we call it $L p$ quasi 2 - normed space $\left(L_{p, 2}\right)$. The set of approximations is a space of feedforward neural networks that is constructed in this paper. Existence and uniqueness of best neural approximation for a function from $L_{p, 2}$ is proved, describing the rate of best approximation in terms of modulus of smoothness.
\end{abstract}

Copyright $(2) 2020$ Institute of Advanced Engineering and Science. All rights reserved.

Corresponding Author:

Hawraa Abbas Almurieb, Department of Mathematics, University of Babylon, Iraq. Email: pure.hawraa.abbas@uobabylon.edu.iq

\section{INTRODUCTION}

The first notes about simultaneous approximation was done by Dunham in [1]. He generated the classical Chebyshev approximation by approximating two continuous functions $f^{+}$and $f^{-}$, with $f^{+}(x) \leq f^{-}(x)$, for all $x \in[a, b]$, simultaneously. He also proved that his simultaneous approximation is equivalent to the classical one function Chebyshev approximation when $f^{+}=f^{-}$.

For more specification, Diaz and Mclaughlin [2] proved that the above problem is equivalent to the problem of approximating $\frac{1}{2}\left|f^{+}+f^{-}\right|$. Also approximating two appropriate functions simultaneously is equivalent to approximating one function by elements of a certain set $\mathbb{S}$. Moreover, they defined the best simultaneous approximation $\mathcal{S}$ to the set $\mathbb{S}$ in [3] as follow

$$
\inf _{s \in \mathbb{S}} \sup _{f \in \mathcal{F}}\|f-s\|=\sup _{f \in \mathcal{F}}\|f-\mathcal{S}\|,
$$

where $\mathcal{F}$ is a set of uniformly bounded functions on $[a, b]$ and $\mathbb{S}$ is a set of functions on $[a, b]$. They proved that $\mathcal{S}$ is equivalent to the best simultaneous approximation of two functions. The set $\mathcal{F}$ varies among researchers, it is $C[a, b]$ in [4] and [5], the space of uniformly bounded functions in [6], Banach space in [7], weighted space [8], Lp spaces [4] or 2-normed space as in [9-16].

The 2-normed space was firstly defined by Gahler in his paper [9], and then generalized by Iseki in his paper [17]. This space provides a tool to deal with 2-structures. For the same porpuse, others defined quasi-normed, quasi-(2;p)-normed space and generalized each one (see [18, 19]). First, we define the 2-normed space generally from [1]

Definition (1) A norm $\|\because\|: X \times X \rightarrow R^{+}$is 2-norm on $\mathrm{X}$ if it satisfies the following conditions:

[C1] $\left\|x_{1}, x_{2}\right\|=0$, if and only if $x_{1}, x_{2}$ are linearly dependent from $\mathrm{X}$. .

[C2] $\left\|x_{1}, x_{2}\right\|=\left\|x_{2}, x_{1},\right\|$, for all $x_{1}, x_{2}$ from $\mathrm{X}$. 
[C3] $\left\|\alpha x_{1}, x_{2}\right\|=|\alpha|\left\|x_{1}, x_{2}\right\|$, for all $\alpha \in R$ and $x_{1}, x_{2} \in \mathrm{X}$

[C4] $\left\|x_{1}+x_{2}, z\right\| \leq\left\|x_{1}, z\right\|+\left\|x_{2}, z\right\|$, for all $x_{1}, x_{2}, z \in X$. condition

The space $(X,\|\because\|)$ is called 2-normed space. Later, Park [18] substitute [C4] with the following

$[\mathrm{C} 4 *]\left\|x_{1}+x_{2}, z\right\| \leq C\left\{\left\|x_{1}, z\right\|+\left\|x_{2}, z\right\|\right\}$, for all $x_{1}, x_{2}, z \in X$.

In this paper, we deal with 2 -normed space with a special definition that deals with Lebesgue-integrable space

$$
L_{p}[a, b]=\left\{f: \int_{a}^{b}|f(x)|^{p} d x<\infty\right\}
$$

Through this paper, we refer $L_{p, 2}$ to the space $L_{p}[a, b] \times L_{p}[a, b]$ in the following manner

$$
L_{p, 2}[a, b]=\left\{f: \int_{a}^{b}|f(x) \varphi(x)|^{p} d x<\infty, \text { for every } \varphi \in L_{p}\right\}
$$

Together with the non-neagative function $\|\cdot, \cdot\|_{p}$ over the vector space $L_{p, 2}$ as follow

$$
\|f, g\|_{p}=\left(\int_{a}^{b}|f(x) g(x)|^{p} d x\right)^{1 / p},
$$

for any function $f$ and $g$ from $L_{p, 2}$. The space $L_{p, 2}[a, b]$ is a 2-normed space since it satisfies the following conditions

[C1] $\|f, g\|_{p}=0$, if and only if $f$ and $g$ are linearly dependent functions from $L_{p, 2}$.

[C2] $\|f, g\|_{p}=\|g, f\|_{p}$, for all $f$ and $g$ from $L_{p, 2}$.

[C3] $\|\alpha f, g\|_{p}=|\alpha|\|f, g\|_{p}$, for all $\alpha \in R$ and $f, g \in L_{p, 2}$.

[C4] $\|f+g, \varphi\|_{p} \leq C\left\{\|f, \varphi\|_{p}+\|g, \varphi\|_{p}\right\}$, for all $f, g, \varphi \in L_{p, 2}$.

The space $L_{p, 2}[a, b]$ is a 2-normed space since it satisfies the conditions in Definition(1).

[C1] Let $f, g$ be two linearly dependent functions from $L_{p, 2}$, with $f \neq g$ iff $\langle f, g\rangle=0$, iff $\|f, g\|_{p}=0$.

[C2] By (2), we have $\|f, g\|_{p}=\|g, f\|_{p}$.

[C3] Let $\alpha \in R$, then $\|\alpha f, g\|_{p}=\left(\int_{a}^{b}|\alpha f(x) g(x)|^{p} d x\right)^{\frac{1}{p}}=|\alpha|\left(\int_{a}^{b}|f(x) g(x)|^{p} d x\right)^{\frac{1}{p}},=|\alpha|\|f, g\|_{p}$,

[C4] Let $f, g \in L_{p, 2}$, since $0<p<1$, then there exists $C>0$ satisfies

$$
\begin{aligned}
& \|f+g, \varphi\|_{p}=\left(\int_{a}^{b}|(f+g)(x) \varphi(x)|^{p} d x\right)^{\frac{1}{p}} \\
& \leq C\left\{\left(\int_{a}^{b}|f(x) \varphi(x)|^{p} d x\right)^{\frac{1}{p}}+\left(\int_{a}^{b}|g(x) \varphi(x)|^{p} d x\right)^{\frac{1}{p}}\right\} \\
& =C\left\{\|f, \varphi\|_{p}+\|g, \varphi\|_{p}\right\} .
\end{aligned}
$$

To continue our investigation for a neural best approximation, we need the following definitions that are related to convergence sequences of functions from $L_{p, 2}$.

if

Definition(2) A sequence of functions $\left\{f_{n}\right\}_{n \geq 1}$ from $L_{p, 2}$ is said to be Cauchy Sequence if and only

$$
\lim _{n, m \rightarrow \infty}\left\|f_{n}-f_{m}, \varphi_{1}\right\|_{p}=0
$$

and

$$
\lim _{n, m \rightarrow \infty}\left\|f_{n}-f_{m}, \varphi_{2}\right\|_{p}=0
$$

for some independent functions $\varphi_{1}, \varphi_{2} \in L_{p, 2}$.

Definition(3) A sequence of functions $\left\{f_{n}\right\}_{n \geq 1}$ from $L_{p, 2}$ is said to be convergent to some $f \in L_{p, 2}$ if and only if

$$
\lim _{n, m \rightarrow \infty}\left\|f_{n}-f, \varphi\right\|_{p}=0,
$$


for all $\varphi \in L_{p, 2}$. The following definitions give some useful properties to the space $L_{p, 2}$, that we need later in the main results.

Definition(4) The space $L_{p, 2}$ is said to be complete if and only if every Cauchy sequence $\left\{f_{n}\right\}_{n \geq 1}$ from $L_{p, 2}$ converges to a function that belongs to $L_{p, 2}$. To measure the degree of best approximation, we define the modulus of smoothness in $L_{p, 2}$ as follow

Definition(5) Let $f \in L_{p, 2}$, then the $k$ th symmetric difference of $f$ is given by

$$
\Delta_{h}^{k}(f, x,[a, b])=\left\{\begin{array}{cl}
\sum_{i=0}^{k}\left(\begin{array}{c}
k \\
i
\end{array}\right)(-1)^{k-i} f\left(x-\frac{k h}{2}+i h\right), & x \pm \frac{k h}{2} \in[a, b] \\
0 & \text { o.w. }
\end{array}\right.
$$

So the $k$ th modulus of smoothness of $f$ is given by

$$
\omega_{k}(f, \varphi, \delta,[a, b])_{p}=\sup _{0<h \leq \delta}\left\|\Delta_{h}^{k}(f ;), \varphi\right\|_{p^{\prime}}
$$

for some $\delta \geq 0$.

\section{CONSTRUCTION OF FNN WITH RELU ACTIVATION FUNCTION}

We have to talk about the set of approximation. Choosing the target approximation space is as much important as choosing the function space. It is related to the applicable properties and the accurate results to each space. Moreover, sometimes it is preferred to replace a certain function by its approximation from some vital space. Scientists approximate functions by polynomials, wavelets, splines and neural networks. For the wide usage of neural networks and their ability to solve problems from different fields (see [21-38]) a set of functions from Lp space is approximated by neural networks in this work. Many papers contains this topic widely, we mention some of them in the references below (see [39-47]).

Let the approximation neural operator

$$
\mathcal{N}=\sum_{i=1}^{n} c_{i} \mathcal{R}\left(w_{i} x+\vartheta_{i}\right)
$$

where

$$
\mathcal{R}(x)=x^{+}=\max (0, x)=\left\{\begin{array}{ll}
0, & x \leq 0 \\
x, & x>0
\end{array}\right\},
$$

is the Relu activation function. For its simplicity and efficiency, scientists use Relu function to activate the neural network. In comparison with other activation functions, it gives faster and more acceptable results, it solves the problem of vanishing gradient that most activation functions suffer from. In the field of function approximation, [48-50] are some papers that dealt with neural approximation with Relu activation function. Now, we are ready to discuss the essential point in this paper. Here is the definition of the best simultaneous approximation of the set $L_{p, 2}$ by elements of $\aleph$ under the norm (2).

Definition(6) The simultaneous best approximation of a subset $\mathcal{F}$ of $L_{p, 2}$ is $N^{*} \in \mathcal{N}$ in the expression

$$
\inf _{N \in \mathbb{K}}\left\{\sup _{f \in \mathcal{F}}\|f-N, \varphi\|_{p}\right\}=\sup _{f \in \mathcal{F}}\left\|f-N^{*}, \varphi\right\|_{p}
$$

In the next section, we construct our neural approximation of type (5) simultaneously to $L_{p, 2}$.

\section{EXISTENCE THEOREM}

Let $f \in L_{p, 2}$, then there exists a FNN of the form:

$$
\mathcal{N}_{n}=\sum_{i=1}^{n} c_{i} \mathcal{R}\left(w_{i} x+\vartheta_{i}\right)
$$

where $\mathcal{R}$ is the Relu activation function on $[a, b]$ and the parameters $c_{i}, w_{i}$, and $\vartheta_{i}$ are chosen as follow: 


$$
\begin{aligned}
& w_{i}=-2 \frac{h n}{|b-a|^{\prime}} \\
& \vartheta_{i}=\frac{h n}{|b-a|}\left(2 a+(2 i-1) \frac{b-a}{n}\right), \\
& c_{0}=f(a)-\sum_{i=1}^{n} c_{i} \mathcal{R}\left(w_{i} a+\vartheta_{i}\right), \\
& c_{i}=\frac{1}{2 b} \sum_{i=0}^{k}\left(\begin{array}{l}
k \\
i
\end{array}\right)(-1)^{k-i} f\left(x-\frac{k h}{2}+i h\right),
\end{aligned}
$$

where $h=\frac{b}{2 n}$.

\section{Proof:}

Since $\mathcal{R}(x)=x^{+}, \forall x \in[a, b]$, then by (6), sup $|\mathcal{R}(x)|=b$.

Let the partition $a<x_{1}<x_{2}<\cdots<x_{n}=b$, such that for all $1 \leq i \leq n$, and let $x_{i}=a+i \frac{b-a}{n}$,

Choosing $c_{0}=f(a)-\sum_{i=1}^{n} c_{i} \mathcal{R}\left(w_{i} a+\vartheta_{i}\right)$, gives the guaranty that $f(a)=\mathcal{N}_{n}(a)$.

For all $x \in[a, b]$, there is $j \in \mathrm{N}, 0 \leq j \leq n$, such that $x \in\left[x_{j-1}, x_{j}\right]$, and that

$$
\begin{aligned}
& \mathcal{N}_{n}(x)=f(a)+\sum_{i=1}^{n} \frac{1}{2 b} \sum_{l=0}^{k}\left(\begin{array}{l}
k \\
l
\end{array}\right)(-1)^{k-l} f\left(x-\frac{k h}{2}+l h\right)\left[\mathcal{R}\left(w_{i} x+\vartheta_{i}\right)-\mathcal{R}\left(w_{i} a+\vartheta_{i}\right)\right] \\
& =f(a)+\sum_{i=1}^{j-1} \frac{1}{2 b} \sum_{l=0}^{k}\left(\begin{array}{c}
k \\
l
\end{array}\right)(-1)^{k-l} f\left(x-\frac{k h}{2}+l h\right)\left[\mathcal{R}\left(w_{i} x+\vartheta_{i}\right)-\mathcal{R}\left(w_{i} a+\vartheta_{i}\right)\right] \\
& +\frac{1}{2 b} \sum_{l=0}^{k}\left(\begin{array}{c}
k \\
l
\end{array}\right)(-1)^{k-l} f\left(x_{j}-\frac{k h}{2}+l h\right)\left[\mathcal{R}\left(w_{i} x+\vartheta_{i}\right)-\mathcal{R}\left(w_{i} a+\vartheta_{i}\right)\right] \\
& +\sum_{i=j+1}^{n} \frac{1}{2 b} \sum_{l=0}^{k}\left(\begin{array}{l}
k \\
l
\end{array}\right)(-1)^{k-l} f\left(x-\frac{k h}{2}+l h\right)\left[\mathcal{R}\left(w_{i} x+\vartheta_{i}\right)-\mathcal{R}\left(w_{i} a+\vartheta_{i}\right)\right] \\
& =f(a)+S_{1}+S_{2}+S_{3}
\end{aligned}
$$

To estimate $\left|\mathcal{R}\left(w_{i} x+\vartheta_{i}\right)-\mathcal{R}\left(w_{i} a+\vartheta_{i}\right)\right|$, we have two cases. For $i>j$, we have, $x \leq x_{j} \leq x_{i-1}$, so by monotonicity of $\mathcal{R}$ and our choices of the parameters $w_{i}, \vartheta_{i}$ and $x_{i}$, we get

\section{Case(1)}

$$
\begin{aligned}
& 0<\mathcal{R}\left(w_{i} x+\vartheta_{i}\right)-\mathcal{R}\left(w_{i} a+\vartheta_{i}\right) \\
& \leq \mathcal{R}\left(w_{i} x_{j}+\vartheta_{i}\right)-\mathcal{R}\left(w_{i} a+\vartheta_{i}\right) \\
& \leq \mathcal{R}\left(w_{i} x_{i-1}+\vartheta_{i}\right)-\mathcal{R}\left(w_{i} a+\vartheta_{i}\right) \\
& =\mathcal{R}(h)-\mathcal{R}(2 h i-h) \\
& =-2 h i=-\frac{b i}{n} .
\end{aligned}
$$

\section{Case(2)}

For $i<j$, we have, $x_{i} \leq x_{j-1} \leq x$, then

$$
\begin{aligned}
& \mathcal{R}\left(w_{i} x+\vartheta_{i}\right)-\mathcal{R}\left(w_{i} a+\vartheta_{i}\right) \\
\geq & \mathcal{R}\left(w_{i} x_{i}+\vartheta_{i}\right)-\mathcal{R}\left(w_{i} x_{i-1}+\vartheta_{i}\right) \\
= & \mathcal{R}(-h)-\mathcal{R}(h) \\
= & -h=-\frac{b}{2 n} .
\end{aligned}
$$


For the two cases, we conclude that

$\left|\mathcal{R}\left(w_{i} x+\vartheta_{i}\right)-\mathcal{R}\left(w_{i} a+\vartheta_{i}\right)\right| \leq h=\frac{b}{2 n}$

Now, we are ready to estimate $S_{1}, S_{2}$ and $S_{3}$

$\left|S_{1}\right| \leq \frac{1}{2 b} \sum_{i=1}^{j-1} \sum_{l=0}^{k}\left(\begin{array}{l}k \\ l\end{array}\right)(-1)^{k-l}\left|f\left(x-\frac{k h}{2}+l h\right)\right|\left|\mathcal{R}\left(w_{i} x+\vartheta_{i}\right)-\mathcal{R}\left(w_{i} a+\vartheta_{i}\right)\right|$

$\leq \frac{1}{4} \Delta_{h}^{k}(f, x,[a, b])$

$\left|S_{2}\right| \leq \frac{1}{2 b} \sum_{l=0}^{k}\left(\begin{array}{l}k \\ l\end{array}\right)(-1)^{k-l}\left|f\left(x_{j}-\frac{k h}{2}+l h\right)\right|\left|\mathcal{R}\left(w_{i} x+\vartheta_{i}\right)-\mathcal{R}\left(w_{i} a+\vartheta_{i}\right)\right|$

$\leq \frac{1}{4} \Delta_{h}^{k}(f, x,[a, b])$

$\left|S_{3}\right| \leq \frac{1}{2 b} \sum_{i=j+1}^{n} \sum_{l=0}^{k}\left(\begin{array}{c}k \\ l\end{array}\right)(-1)^{k-l}\left|f\left(x-\frac{k h}{2}+l h\right)\right|\left|\mathcal{R}\left(w_{i} x+\vartheta_{i}\right)-\mathcal{R}\left(w_{i} a+\vartheta_{i}\right)\right|$

$\leq \frac{1}{4} \Delta_{h}^{k}(f, x,[a, b])$

Finally, let $\in L_{p, 2}$, then

$\left\|\mathcal{N}_{n}(x)-f(x), \varphi\right\|_{p}^{p} \leq \int_{a}^{b}\left|\mathcal{N}_{n}(x)-f(x)\right|^{p}|\varphi(x)|^{p} d x$

$\leq \frac{1}{2 b} \sum_{i=1}^{n}\left[\mathcal{R}\left(w_{i} x+\vartheta_{i}\right)-\mathcal{R}\left(w_{i} a+\vartheta_{i}\right)\right] \int_{a}^{b} \sum_{l=1}^{k}\left(\begin{array}{l}k \\ l\end{array}\right)(-1)^{k-l}\left|f\left(x-\frac{k h}{2}+l h\right)\right|^{p}|\varphi(x)|^{p} d x$

$\leq \frac{3}{4} \omega_{k}\left(f, \varphi, \frac{1}{n}\right)_{p}$

\section{UNIQUENESS THEOREM}

The simultaneous best approximation $N^{*} \in \aleph$ of a subset $F$ of $L_{p, 2}$ is unique.

\section{Proof:}

To prove that $N^{*} \in \mathcal{N}$ is unique, suppose that $\mathrm{N}_{1}, \mathrm{~N}_{2} \in \mathcal{N}$ be two simultaneous approximations to $F$, then by Definition(3)

$$
\lim _{n \rightarrow \infty}\left\|N_{1}-f, \varphi\right\|_{p}=0,
$$

and

$\lim _{n \rightarrow \infty}\left\|N_{2}-f, \varphi\right\|_{p}=0$,

So by condition [C4*] of Definition(1), there exists $k \geq 1$,

$\left\|\mathrm{N}_{1}-\mathrm{N}_{2}, \varphi\right\|_{p} \leq k\left(\left\|\mathrm{~N}_{1}-f, \varphi\right\|_{p}+\left\|\mathrm{N}_{2}-f, \varphi\right\|_{p}\right)$

By taking limits to both sides as $\mathrm{n}$ tends to infinity, then for all $\varphi \in \mathrm{L}_{p, 2}$

$\lim _{n \rightarrow \infty}\left\|\mathrm{N}_{1}-\mathrm{N}_{2}, \varphi\right\|_{p}=0$,

So $\mathrm{N}_{1}=\mathrm{N}_{2}$, and the best simultaneous approximation to $F$ out of $\mathrm{N}$ is unique. 


\section{CONCLUSION}

Simultaneous approximation in the $L_{p, 2}$ space is defined in details in this paper. Construction of neural networks with rectified activation function that approximates a subset of $L_{p, 2}$ simultaneously is obtained too. In spite of its various applications, it gives accurate results that depends on modulus of smoothness. It would be interesting to discuss vital applications in 2-structure spaces for the constructed neural network.

\section{REFERENCES}

[1] C. B. Dunham, "Simultaneous Chebyshev Approximation of Functions on an Interval," Proc. Am. Math. Soc., vol. 18 , no. 3, p. $472,1967$.

[2] J. B. Diaz and H. W. Mclaughlin, "On Simultaneous Chebyshev Approximation and Chebyshev Approximation with an Additive Weight," J. Approx. Theory, vol. 71, no. 6, pp. 68-71, 1972.

[3] J. B. Diaz and H. W. Mclaughlin, "Simultaneous approximation of a set of bounded real functions," Math. Comput., vol. 23, no. 107, pp. 583-594, 1969.

[4] F. E. Levis, "Interpolation and best simultaneous approximation," 2017.

[5] C. Li and G. A. Watson, "On nonlinear simultaneous Chebyshev approximation problems," J. Math. Anal. Appl., vol. 288, no. 1, pp. 167-181, 2003.

[6] Ş. Atacik, "Simultaneous approximation of a uniformly bounded set of real valued functions," J. Approx. Theory, vol. 45, no. 2, pp. 129-132, 1985.

[7] P. D. Milan, "On Best Simultaneous Approximation in Normed Linear Spaces," J. Approx. Theory, vol. 238, pp. 223-238, 1977.

[8] A. A. L. I. Hussein, "Best simultaneous approximation in weighted space Best simultaneous approximation in weighted space," 2019.

[9] S. Gahler, "Lineare 2-Normierte Räume," Math. Nachrichten, vol. 28, pp. 1-43, 1964.

[10] S. Elumalai and R. Vijayaragavan, "Characterizations of best approximations in linear 2-normed spaces," Gen. Math., vol. 17, no. 3, pp. 141-160, 2009.

[11] A. Mehmet, "The best simultaneous approximation in linear 2-normed spaces," Proc. Jangjeon Math. Soc., vol. 15, no. 4, pp. 415-422, 2012.

[12] M. Acikgoz, "The best simultaneous approximation in linear 2-normed spaces," 2014.

[13] M. Acikgoz, "Approximation in generalized 2-normed spaces," 2014.

[14] S. Cobzas and R. Academy, "Extension of bilinear functionals and best approximation in 2-normed spaces," 2017.

[15] M. Iranmanesh and F. Soleimany, “2-NORMED SPACES," vol. 46, no. 1, pp. 207-215, 2016.

[16] A. Kundu and T. B. Sk, "On metrizability and normability of 2-normed spaces," Math. Sci., vol. 13, no. 1, pp. 69-77, 2019.

[17] K. Iseki, "Mathematics on 2-normed spaces," Korean Math. Soc., vol. 13, pp. 127-136, 1976.

[18] C. Park, "Generalized quasi-banach spaces and quasi- (2;p)-Normed Spaces," J. Chungcheong Math. Soc., vol. 19, no. 2, pp. 197-206, 2006

[19] K. Kikina, et al., "Quasi-2-Normed Spaces and Some Fixed Point Theorems," vol. 474, no. 2, pp. 469-474, 2016.

[20] C. Park, "Generalized quasi-Banach spaces and quasi-(2, p)- normed spaces," J. Chungcheong Math. Soc., vol. 19, no. 2, pp. 197-206, 2006.

[21] L. E. Aik, et al., "An improved radial basis function networks based on quantum evolutionary algorithm for training nonlinear datasets," IAES Int. J. Artif. Intell., vol. 8, no. 2, pp. 120-131, 2019.

[22] A. S. Takialddin, et al., "Overview of model free adaptive (MFA) control technology," IAES Int. J. Artif. Intell., vol. 7, no. 4, pp. 165-169, 2018.

[23] V. Kathirvel, et al., "Hybrid imperialistic competitive algorithm incorporated with hopfield neural network for robust 3 satisfiability logic programming," IAES Int. J. Artif. Intell., vol. 8, no. 2, pp. 144-155, 2019.

[24] M. Rhanoui, et al., "Forecasting financial budget time series: Arima random walk vs 1stm neural network," IAES Int. J. Artif. Intell., vol. 8, no. 4, pp. 317-327, 2019.

[25] S. Ibrahim, et al., "Optimization of artificial neural network topology for membrane bioreactor filtration using response surface methodology," IAES Int. J. Artif. Intell., vol. 9, no. 1, pp. 117-125, 2020.

[26] S. I. Abdullahi, et al., "Intelligent flood disaster warning on the fly: Developing IoT-based management platform and using 2-class neural network to predict flood status," Bull. Electr. Eng. Informatics, vol. 8, no. 2, pp. 706-717, 2019.

[27] S. Verma, et al., "ANN based method for improving gold price forecasting accuracy through modified gradient descent methods," IAES Int. J. Artif. Intell., vol. 9, no. 1, pp. 46-57, 2020.

[28] I. Hachchane, et al., "Large-scale image-to-video face retrieval with convolutional neural network features," IAES Int. J. Artif. Intell., vol. 9, no. 1, pp. 40-45, 2020.

[29] P. R. Iyer, et al., "Adaptive real time traffic prediction using deep neural networks," IAES Int. J. Artif. Intell., vol. 8, no. 2, pp. 107-119, 2019.

[30] W. N. W. Md Adnan, et al., "Development of option c measurement and verification model using hybrid artificial neural network-cross validation technique to quantify saving," IAES Int. J. Artif. Intell., vol. 9, no. 1, pp. 25-32, 2020.

[31] S. B. Jadhav, et al., "Convolutional neural networks for leaf image-based plant disease classification," IAES Int. J. Artif. Intell., vol. 8, no. 4, pp. 328-341, 2019. 
[32] S. Barhmi and O. El Fatni, "Hourly wind speed forecasting based on support vector machine and artificial neural networks," IAES Int. J. Artif. Intell., vol. 8, no. 3, pp. 286-291, 2019.

[33] N. Mahmod, et al., "Modelling and control of fouling in submerged membrane bioreactor using neural network internal model control," IAES Int. J. Artif. Intell., vol. 9, no. 1, pp. 100-108, 2020.

[34] L. E. Aik, et al., "An improved radial basis function networks in networks weights adjustment for training realworld nonlinear datasets," IAES Int. J. Artif. Intell., vol. 8, no. 1, pp. 63-76, 2019.

[35] H. A. Rahim, et al., "Exploration on digital marketing as business strategy model among malaysian entrepreneurs via neurocomputing," IAES Int. J. Artif. Intell., vol. 9, no. 1, pp. 18-24, 2020.

[36] H. Ohmaid, et al., "Iris segmentation using a new unsupervised neural approach," IAES Int. J. Artif. Intell., vol. 9, no. 1 , pp. 58-64, 2020.

[37] A. Sarkar, "Multilayer neural network synchronized secured session key based encryption in wireless communication," IAES Int. J. Artif. Intell., vol. 8, no. 1, pp. 44-53, 2019.

[38] M. S. Gaya, et al., "Estimation of water quality index using artificial intelligence approaches and multi-linear regression," IAES Int. J. Artif. Intell., vol. 9, no. 1, pp. 126-134, 2020.

[39] G. Cybenko, "Continuous Valued Neural Networks: Approximation Theoretic Results," in Computer Science and Statistics: proceedings of the 20th Symposium on the Interface, pp. 174-183, 1988.

[40] T. Chen and R. Chen, "Approximation Capability to Functions of Several Variables, Nonlinear Functionals and Operators by Radial Basis Function Neural Networks," IEEE Trans. Neural Networks, vol. 6, no. 4, pp. 904-910, 1995.

[41] Z. Zhang, et al., "The new approximation operators with sigmoidal functions," J. Appl. Math. Comput, vol. 42, no. 1-2, pp. 455-468, 2013.

[42] E. S. Bhaya, "Neural Network Trigonometric Approximation," no. 9, pp. 2395-2399, 2016.

[43] H. A. Almurieb, "Simultaneous Approximation of order m by Artificial Neural Network," vol. 56, no. 4, 2017.

[44] E. S. Bhaya and H. A. Almurieb, "Neural Network Trigonometric Approximation," J. Univ. Babylon, vol. 26, no. 1, pp. 2395-2399, 2016.

[45] E. S. Bhaya and M. A. Kareem, "Whitney Multiapproximation,” J. Univ. Babylon Pure Appl. Sci., vol. 24, no. 9, pp. 385-403, 2018.

[46] E. S. Bhaya and S. Z. Abdulmunim, "Approximation of Functions in L_P Spaces for $\mathrm{p}<1$, Using Radial Basis Function Neural Networks,” J. Univ. Babylon Pure Appl. Sci., vol. 27, no. 3, pp. 400-405, 2019.

[47] E. Bhaya, "lp approximation using radial basis neural network on ordered space," 2019.

[48] M. Hansson and C. Olsson, "Feedforward neural networks with ReLU activation functions are linear splines," 2017.

[49] H. Montanelli, et al., "Deep ReLU networks overcome the curse of dimensionality for bandlimited functions," 2019.

[50] E. J. S. Dittmer and P. Maass, "Singular Values for ReLU Layers," IEEE Trans. Neural Networks Learn. Syst., no. 2, pp. 1-12, 2019.

\section{BIOGRAPHIES OF AUTHORS}

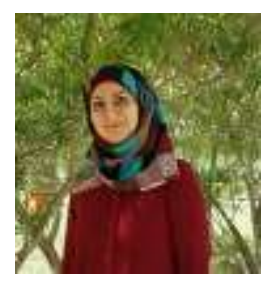

She received her MSc degree in science of mathematics from University of Babylon in 2010 and sh is a PhD student of applied mathematics at University of Baghdad too in 2004. She has been a lecturer at University of Kufa and then University of Babylon in Iraq. Her current research interests include approximation theory, essential approximation, neural networks

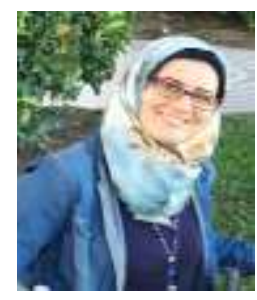

She received her MSc degree in science of mathematics from University of Baghdad 1999 and her $\mathrm{PhD}$ degree in science of mathematics from University of Baghdad too in 2004. Since then she has been a professor at the University of Babylon in Iraq. She has tens of published papers in research interests include approximation theory, interpolation, and neural networks. 